\title{
Search for Excited Spin-3/2 Neutrinos at LHeC
}

\author{
A. Ozansoy, ${ }^{1}$ V. Ar1, ${ }^{1}$ and V. Çetinkaya ${ }^{2}$ \\ ${ }^{1}$ Department of Physics, Ankara University, Tandogan, 06100 Ankara, Turkey \\ ${ }^{2}$ Department of Physics, Dumlupinar University, Merkez, 43100 Kutahya, Turkey \\ Correspondence should be addressed to A. Ozansoy; aozansoy@science.ankara.edu.tr
}

Received 1 June 2016; Revised 21 September 2016; Accepted 19 October 2016

Academic Editor: Andrea Coccaro

Copyright (C) 2016 A. Ozansoy et al. This is an open access article distributed under the Creative Commons Attribution License, which permits unrestricted use, distribution, and reproduction in any medium, provided the original work is properly cited. The publication of this article was funded by SCOAP ${ }^{3}$.

\begin{abstract}
We study the potential of the next ep collider, namely, LHeC, with two options $\sqrt{s}=1.3 \mathrm{TeV}$ and $\sqrt{s}=1.98 \mathrm{TeV}$, to search for excited spin-1/2 and spin-3/2 neutrinos. We calculate the single production cross-section of excited spin-1/2 and spin-3/2 neutrinos according to their effective currents describing their interactions between gauge bosons and SM leptons. We choose the $v^{\star} \rightarrow e W$ decay mode of excited neutrinos and $W \rightarrow j j$ decay mode of $W$-boson for the analysis. We put some kinematical cuts for the final state detectable particles and plot the invariant mass distributions for signal and the corresponding backgrounds. In order to obtain accessible limits for excited neutrino couplings, we show the $f-f^{\prime}$ and $c_{i V}-c_{i A}$ contour plots for excited spin-1/2 and excited spin-3/2 neutrinos, respectively.
\end{abstract}

\section{Introduction}

The Standard Model (SM) of the particle physics agrees with experimental results from the operating colliders. The first run of the Large Hadron Collider (LHC) brought the expected Higgs boson discovery, so a crucial part of the $\mathrm{SM}$ has been completed. But there is still no satisfying explanation of the three-family structure of leptons and quarks and their mass hierarchy. An attractive explanation is lepton and quark compositeness [1-3]. In composite models, known leptons and quarks have a substructure characterized by an energy scale called the compositeness scale, $\Lambda$. A natural consequence of compositeness is the occurrence of excited states [4-7]. Phenomenologically, an excited lepton can be regarded as a heavy lepton sharing the same leptonic quantum number with the corresponding SM lepton. If leptons present composite structures, they can be considered as spin- $1 / 2$ bound states containing three spin- $1 / 2$ or spin- $1 / 2$ and spin- 0 subparticles. Bound states of spin-3/2 leptons are also possible with three spin-1/2 [1-3] or spin-1/2 and spin-1 subparticles in the framework of composite models [8]. The motivations for spin-3/2 particles come from two different scenarios; spin-3/2 leptons appear in composite models [913 ] and a spin-3/2 gravitino is the superpartner of graviton in supergravity [14]. Theories beyond the Standard Model that contain exotic particles are discussed in [15-19].

Both excited spin-1/2 and spin-3/2 neutrinos can be produced at future high energy lepton, hadron, and leptonhadron colliders. Elaborate studies on excited spin-1/2 neutrinos can be found in [20-30]. Also, one can find excited spin$1 / 2$ neutrino production by ultra-high energy neutrinos in [31] and the impact of excited spin-1/2 neutrinos on $\nu \bar{\nu} \rightarrow \gamma \gamma$ process in [32].

The mass limit for excited spin- $1 / 2$ neutrinos obtained from their pair production $\left(e^{+} e^{-} \rightarrow v^{\star} v^{\star}\right.$ process $)$ by L3 Collaboration at $\sqrt{s}=189-209 \mathrm{GeV}$, assuming $f=-f^{\prime}$, where $f$ and $f^{\prime}$ are the new couplings determined by the composite dynamics, is $m^{\star}>102.6 \mathrm{GeV}$ [33]. Assuming $f=$ $f^{\prime}$ and $f / \Lambda=1 / m^{\star}$, for single production of excited spin-1/2 neutrino in $e p$ collisions taking into account all the decay channels, the H1 Collaboration sets the exclusion limit for the mass range of excited neutrino $m^{\star}>213 \mathrm{GeV}$ at $95 \%$ C.L. [34]. Recently, a search was performed by the ATLAS Collaboration taking into account pair production of excited spin$1 / 2$ neutrinos either through contact or gauge-mediated interactions and their decay proceeds via the same mechanism. Considering events with at least three charged leptons with $\Lambda=m^{\star}$, with $f=f^{\prime}=1$ and with an integrated luminosity of 
TABLE 1: Branching ratios and total decay width of excited spin-1/2 neutrinos for $f=-f^{\prime}=1\left(f=f^{\prime}=1\right)$. Here it is taken as $\Lambda=m^{\star}$.

\begin{tabular}{lcccc}
\hline$m^{\star}(\mathrm{GeV})$ & $\Gamma(\mathrm{GeV})$ & $\% \mathrm{BR}\left(\nu^{\star} \rightarrow \nu \gamma\right)$ & $\% \mathrm{BR}\left(\nu^{\star} \rightarrow \nu Z\right)$ & $\% \mathrm{BR}\left(\nu^{\star} \rightarrow e W\right)$ \\
\hline 300 & 1.91 & $30.5(0)$ & $10.7(38.3)$ & $58.9(61.7)$ \\
500 & 3.36 & $28.9(0)$ & $11.1(38.9)$ & $60.0(61.1)$ \\
750 & 5.12 & $28.4(0)$ & $11.3(39.0)$ & $60.3(61.0)$ \\
1000 & 6.87 & $28.2(0)$ & $11.3(39.1)$ & $60.4(60.9)$ \\
1500 & 10.35 & $28.1(0)$ & $11.4(39.1)$ & $60.5(60.9)$ \\
2000 & 13.82 & $28.1(0)$ & $11.4(39.1)$ & $60.5(60.9)$ \\
2500 & 17.28 & $28.1(0)$ & $11.4(39.1)$ & $60.5(60.9)$ \\
3000 & 20.75 & $28.1(0)$ & $11.4(39.1)$ & $60.5(60.9)$ \\
\hline
\end{tabular}

$20.3 \mathrm{fb}^{-1}$ of $p p$ collisions at $\sqrt{s}=8 \mathrm{TeV}$, a lower mass limit of $1.6 \mathrm{TeV}$ is obtained for every excited spin-1/2 neutrino flavour [35].

Excited spin-3/2 neutrinos are not as well studied in the litterateur as the spin-1/2. An investigation of the production and decay processes of the single heavy spin- $3 / 2$ neutrino was performed in $[36,37]$. A study of the potential of future high energy $e^{+} e^{-}$linear colliders to probe excited spin-3/2 neutrino signals in different decay modes by considering three phenomenological currents taking into account the corresponding background was done in [8].

Studies are ongoing for the development of a new ep collider, the Large Hadron Electron Collider (LHeC), with an electron beam of $60 \mathrm{GeV}$, to possibly $140 \mathrm{GeV}$, and a proton beam of the LHC [38-41] or in the future the Future Circular Collider lepton-hadron collider (FCC-eh) $[42,43]$. The LHeC is the highest energy lepton-hadron collider under design and is considered as a linac-ring collider. Linac-ring type colliders were proposed in [44] and the physics potentials and advantages of these type lepton-hadron colliders are discussed in $[45,46]$. Latest results for excited neutrino searches coming from the first ep collider HERA have showed that ep colliders are so competitive to $p p$ and $e^{+} e^{-}$colliders and very important for the investigation of beyond SM physics $[34,38-41]$. With the design luminosity of $10^{33} \mathrm{~cm}^{-2} \mathrm{~s}^{-1}$ the $\mathrm{LHeC}$ is intended to exceed the HERA luminosity by a factor of $\sim 100$. So it would be a major opportunity to push forward the investigations done in the LHC.

This work is a continuation of the previous works on excited neutrinos $[8,25]$. In this work, in Section 2 we introduce the phenomenological currents for excited neutrinos and give their decay widths. In Section 3, we consider single production of excited spin-1/2 and spin-3/2 neutrinos at ep colliders. We take into account the signal in $v^{\star} \rightarrow \mathrm{eW}$ decay mode of excited neutrinos as well as corresponding backgrounds at LHeC with $\sqrt{s}=1.3 \mathrm{TeV}$ and $\sqrt{s}=1.98 \mathrm{TeV}$. We plot the invariant mass distributions for single production of excited neutrinos with spin-1/2 and spin-3/2. Last, we plot the contour plots for the excited neutrino couplings to obtain the exclusion limits. Investigation on excited fermions with spin-1/2 takes an important part in the physics program of LHeC $[38,39]$. Although the latest limit for excited spin$1 / 2$ neutrinos set by the ATLAS experiment is high, it is important to examine the excited neutrinos with different spins at high energy lepton-hadron colliders. This work is the only dedicated work which gives the comparative results for both excited spin-1/2 and spin-3/2 neutrinos to comprehend the potential of next ep collider.

\section{Physical Preliminaries}

An excited spin-1/2 neutrino is the lowest radial and orbital excitation according to the classification by $S U(2) \times U(1)$ quantum numbers. Interactions between excited spin-1/2 neutrino and ordinary leptons are of the magnetic transition type [47-49]. The effective current for the interaction between an excited spin-1/2 neutrino, a gauge boson $(V=$ $\gamma, Z, W^{ \pm}$), and the SM lepton is given by

$$
J^{\mu}\left(\frac{1}{2}\right)=\frac{g_{e}}{2 \Lambda} \bar{u}\left(p, \frac{1}{2}\right) i \sigma^{\mu \nu} q_{\nu}\left(1-\gamma_{5}\right) f_{V} u\left(k, \frac{1}{2}\right),
$$

where $\Lambda$ is the new physics scale; $g_{e}$ is electromagnetic coupling constant with $g_{e}=\sqrt{4 \pi \alpha} ; k, p$, and $q$ are the four momentum of the SM lepton, excited spin-1/2 neutrino, and the gauge boson, respectively. $f_{V}$ is the new electroweak coupling parameter corresponding to the gauge boson $V$ and $\sigma^{\mu \nu}=i\left(\gamma^{\mu} \gamma^{\nu}-\gamma^{\nu} \gamma^{\mu}\right) / 2$ with $\gamma^{\mu}$ being the Dirac matrices. An excited neutrino has three possible decay modes, each one of which is related to a vector boson $\gamma, W$, and $Z$. These decay modes are radiative decay $\nu^{\star} \rightarrow \nu \gamma$, neutral weak decay $v^{\star} \rightarrow v Z$, and charged weak decay $\nu^{\star} \rightarrow e W$. Neglecting SM lepton mass we find the decay width of the excited spin-1/2 neutrino as

$$
\begin{aligned}
& \Gamma\left(l^{\star(1 / 2)} \longrightarrow l V\right) \\
& \quad=\frac{\alpha m^{\star 3}}{4 \Lambda^{2}} f_{V}^{2}\left(1-\frac{m_{V}^{2}}{m^{\star 2}}\right)^{2}\left(1+\frac{m_{V}^{2}}{2 m^{\star 2}}\right),
\end{aligned}
$$

where $f_{\gamma}=\left(f-f^{\prime}\right) / 2, f_{Z}=\left(f \cot \theta_{W}+f^{\prime} \tan \theta_{W}\right) / 2$, and $f_{W}=f / \sqrt{2} \sin \theta_{W} ; \theta_{W}$ is the weak mixing angle and $m_{V}$ is the mass of the gauge boson. The couplings $f$ and $f^{\prime}$ are the scaling factors for the gauge couplings of $S U(2)$ and $U(1)$. Unless $f=f^{\prime}$, the electromagnetic interaction of excited neutrino and SM neutrino exists. Branching ratios of excited spin-1/2 neutrino for two choices $f=-f^{\prime}=1$ and $f=f^{\prime}=$ 1 are presented in Table 1 . One may note that for the choice $f=-f^{\prime}=1$ the branching ratio for the $e W$ channel is $\approx 60 \%$. Hence, to choose the $\nu^{\star} \rightarrow e W$ mode for the analysis is more feasible. 
The two phenomenological currents for the interactions between an excited spin-3/2 neutrino, a gauge boson $(V=$ $\left.\gamma, Z, W^{ \pm}\right)$, and the SM lepton are given by

$$
\begin{aligned}
& J_{1}^{\mu}\left(\frac{3}{2}\right)=g_{e} \bar{u}^{\mu}\left(p, \frac{3}{2}\right)\left(c_{1 V}-c_{1 A} \gamma_{5}\right) u\left(k, \frac{1}{2}\right), \\
& J_{2}^{\mu}\left(\frac{3}{2}\right)=\frac{g_{e}}{\Lambda} \bar{u}^{\lambda}\left(p, \frac{3}{2}\right) q_{\lambda} \gamma^{\mu}\left(c_{2 V}-c_{2 A} \gamma_{5}\right) u\left(k, \frac{1}{2}\right),
\end{aligned}
$$

where $u^{\mu}(p, 3 / 2)$ represents the Rarita-Schwinger vectorspinor [50].

Decay widths of excited spin-3/2 neutrinos for the $v^{\star} \rightarrow$ $\nu \gamma$ decay mode for the two currents are given by

$$
\begin{aligned}
& \Gamma_{1}\left(\nu^{\star(3 / 2)} \longrightarrow \nu \gamma\right)=\frac{\alpha}{4}\left(c_{1 V}^{\gamma^{2}}+c_{1 A}^{\gamma^{2}}\right) m^{\star}, \\
& \Gamma_{2}\left(\nu^{\star(3 / 2)} \longrightarrow \nu \gamma\right)=\frac{\alpha}{24}\left(c_{2 V}^{\gamma^{2}}+c_{2 A}^{\gamma^{2}}\right) m^{\star}\left(\frac{m^{\star}}{\Lambda}\right)^{2},
\end{aligned}
$$

and for the neutral and charged weak decay modes $\left(\nu^{\star} \rightarrow \nu Z\right.$ and $\left.v^{\star} \rightarrow e W\right)$, they are given as

$$
\begin{aligned}
\Gamma_{1} & \left(v^{\star(3 / 2)} \longrightarrow l V\right) \\
= & \frac{\alpha}{48}\left(c_{1 V}^{2}+c_{1 A}^{2}\right) m^{\star} \frac{(1-\kappa)^{2}}{\kappa}\left(1+10 \kappa+\kappa^{2}\right), \\
\Gamma_{2} & \left(v^{\star(3 / 2)} \longrightarrow l V\right) \\
& =\frac{\alpha}{48}\left(c_{2 V}^{2}+c_{2 A}^{2}\right) m^{\star}\left(\frac{m^{\star}}{\Lambda}\right)^{2} \frac{(1-\kappa)^{4}}{\kappa}(1+2 \kappa),
\end{aligned}
$$

where $\kappa=\left(m_{V} / m^{\star}\right)^{2}, V=Z, W$, and $l=e, \nu$. Branching ratios and total decay width of excited spin- $3 / 2$ neutrinos with $J_{1}$ and $J_{2}$ are given in Tables 2 and 3 , respectively. Also, total decay width of excited neutrinos as a function of their mass $\left(m^{\star}\right)$ is shown in Figure 1.

\section{Single Production at $e p$ Collider}

The excited spin- $1 / 2$ and spin- $3 / 2$ neutrinos can be produced singly at future ep colliders via $t$-channel $W$ exchange. In our calculations we use the program CALCHEP [51-53]. The Feynman diagrams for the subprocesses $e^{-} q \rightarrow v^{\star} q^{\prime}$ and $e^{-} \overline{q^{\prime}} \rightarrow v^{\star} \bar{q}$ are shown in Figure 2.

Neglecting SM quark masses, the explicit formulas for the differential cross-section of the subprocesses $e^{-} q \rightarrow v^{\star} q^{\prime}$ and $e^{-} \overline{q^{\prime}} \rightarrow v^{\star} \bar{q}$ for the two phenomenological spin-3/2 currents $J_{1}$ and $J_{2}$ are

$$
\begin{array}{r}
\frac{d \sigma_{1}}{d t}=\frac{-2 g_{e}^{2} g_{W}^{2}\left|V_{q q^{\prime}}\right|^{2}\left(-m^{\star 2}+t\right)\left[\left(c_{1 A}^{2}+c_{1 V}^{2}\right) A_{1}\right]}{96 m^{\star 2} \pi s^{2}\left[M_{W}^{4}+t^{2}+M_{W}^{2}\left(-2 t+\Gamma_{W}^{2}\right)\right]}, \\
A_{1}=s(s+t)-m^{\star 2}(s+2 t),
\end{array}
$$

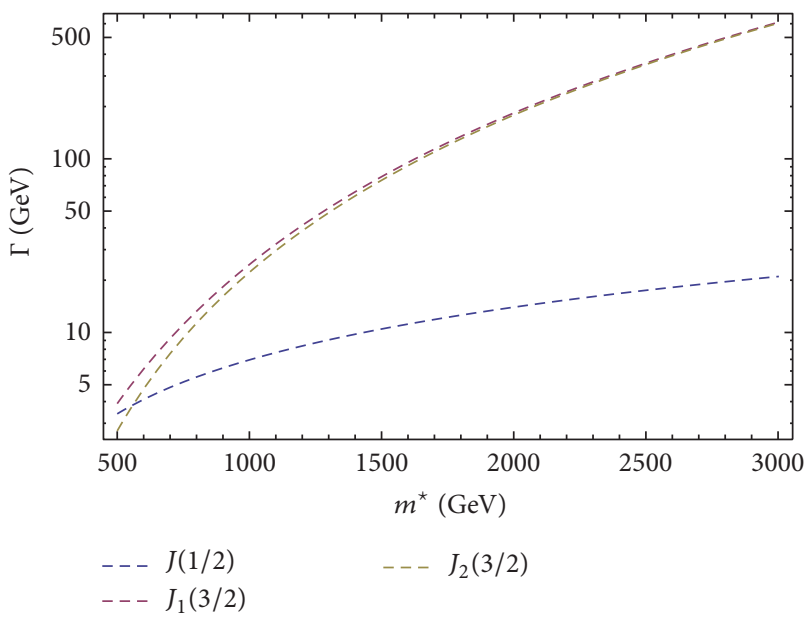

FIGURE 1: Total decay width of excited neutrinos according to their mass. Here, it is taken as $\Lambda=m^{\star}$ and $f=-f^{\prime}=1$ for excited spin-1/2 neutrinos and $c_{i V}=c_{i A}=0.5(i=1,2)$ for excited spin-3/2 neutrinos for the two phenomenological currents.

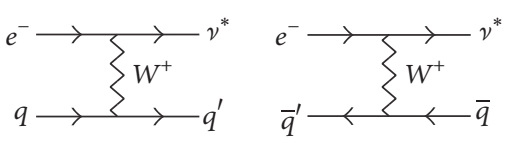

Figure 2: Feynman diagrams.

$$
\begin{array}{r}
\frac{d \sigma_{2}}{d t}=\frac{-2 g_{e}^{2} g_{W}^{2}\left|V_{q q^{\prime}}\right|^{2}\left(m^{\star 2}-t\right)^{2}\left(c_{2 A}^{2}+c_{2 V}^{2}\right) A_{2}}{192 \Lambda^{2} m^{\star 2} \pi s^{2}\left[M_{W}^{4}+t^{2}+M_{W}^{2}\left(-2 t+\Gamma_{W}^{2}\right)\right]} \\
A_{2}=-2 s^{2}-2 s t-t^{2}+m^{\star 2}(2 s+t),
\end{array}
$$

where $V_{q q^{\prime}}$ is the CKM matrix element, $t$ is the Mandelstam variable, and $s$ is the square of center-of-mass energy of the collider. Also, differential cross-section expression for the excited spin- $1 / 2$ neutrino is

$$
\begin{aligned}
& \frac{d \sigma}{d t} \\
& =\frac{g_{e}^{2} g_{W}^{2} f_{W}^{2}\left|V_{q q^{\prime}}\right|^{2} t\left(-m^{\star 4}-2 s(s+t)+m^{\star 2}(2 s+t)\right)}{32 \Lambda^{2} \pi s^{2}\left[M_{W}^{4}+t^{2}+M_{W}^{2}\left(-2 t+\Gamma_{W}^{2}\right)\right]} .
\end{aligned}
$$

Total cross-section as a function of excited neutrino mass is shown in Figure 3 for the center-of-mass energies $\sqrt{s}=$ $1.3 \mathrm{TeV}$ and $\sqrt{s}=1.98 \mathrm{TeV}$.

In our analysis we chose the $\nu^{\star} \rightarrow e W$ mode because of the high branching ratio of the charged current decay channel. $\nu^{\star} \rightarrow \nu \gamma$ and $\nu^{\star} \rightarrow \nu Z$ decay modes will have larger uncertainty because of the missing transverse momentum $\left(\not p_{T}\right)$ due to the neutrino in the final state. We consider the $e p \rightarrow v^{\star} X \rightarrow e^{-} W^{+} X$ process and put some kinematical cuts for the final state detectable particles. We deal with the 

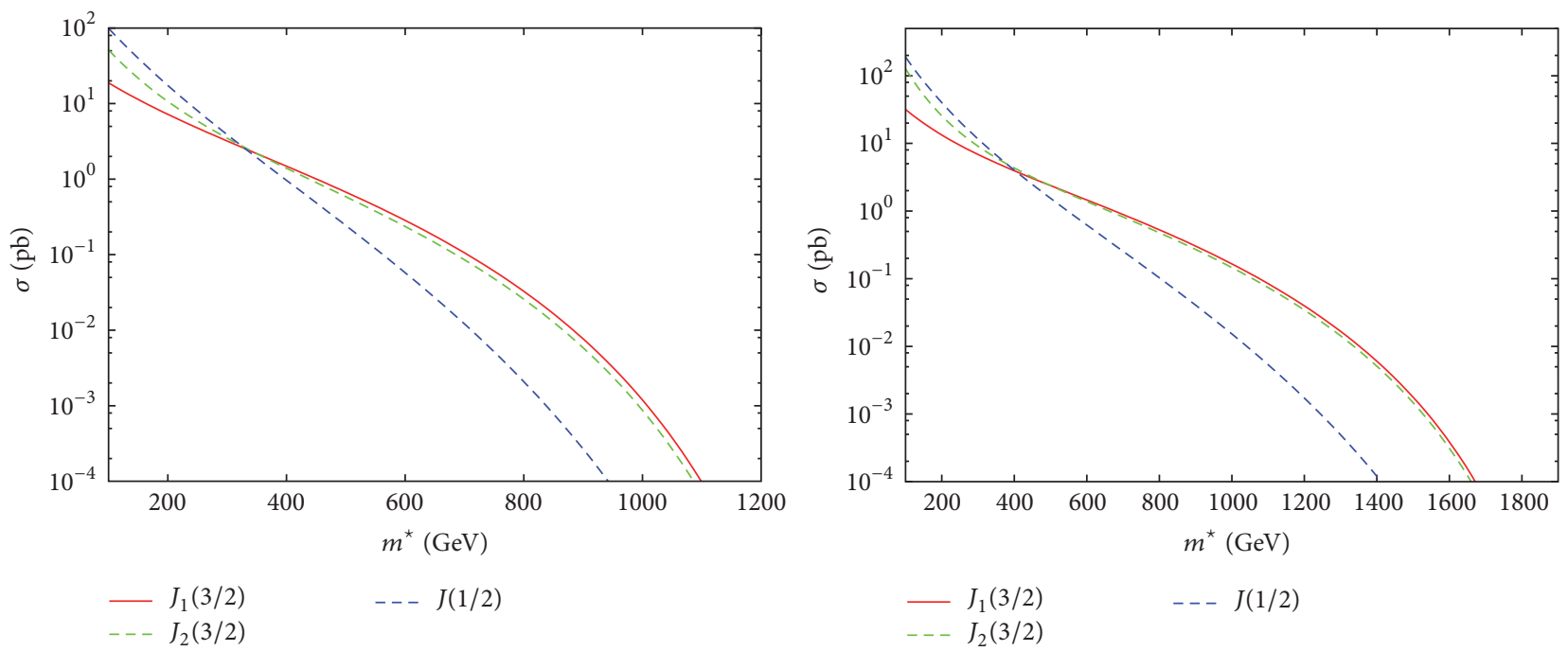

FIgURE 3: Cross-sections for the excited neutrino production with $\Lambda=m^{\star}$ and $f=-f^{\prime}$ for spin-1/2 ones and $c_{i V}=c_{i A}=0.5(i=1,2)$ for spin-3/2 ones at ep collider at $\sqrt{s}=1.3 \mathrm{TeV}$ and $\sqrt{s}=1.98 \mathrm{TeV}$.

TABLE 2: Branching ratios and total decay width of excited spin-3/2 neutrinos with $J_{1}$. Here it is taken as $c_{1 V}=c_{1 A}=0.5$ and $\Lambda=m^{\star}$.

\begin{tabular}{lcccc}
\hline$m^{\star}(\mathrm{GeV})$ & $\Gamma(\mathrm{GeV})$ & $\% \mathrm{BR}\left(\nu^{\star} \rightarrow \nu \gamma\right)$ & $\% \mathrm{BR}\left(\nu^{\star} \rightarrow \nu Z\right)$ & $\% \mathrm{BR}\left(\nu^{\star} \rightarrow e W\right)$ \\
\hline 300 & 1.21 & 24.0 & 34.4 & 41.6 \\
500 & 3.89 & 12.5 & 39.0 & 48.5 \\
750 & 11.11 & 6.5 & 41.2 & 52.3 \\
1000 & 24.61 & 3.9 & 42.1 & 54.0 \\
1500 & 78.89 & 1.8 & 42.8 & 55.3 \\
2000 & 183.50 & 1.1 & 43.1 & 55.9 \\
2500 & 355.20 & 0.7 & 43.2 & 56.1 \\
3000 & 611.00 & 0.5 & 43.3 & 56.2 \\
\hline
\end{tabular}

subprocess $e^{-} q\left(\overline{q^{\prime}}\right) \rightarrow W^{+} e^{-} q^{\prime}(\bar{q})$ and impose the acceptance cuts

$$
\begin{aligned}
p_{T}^{e, q} & >20 \mathrm{GeV} \\
\left|\eta^{e, q}\right| & <2.5 .
\end{aligned}
$$

Feynman diagrams for the $e^{-} q \rightarrow e^{-} W^{+} q^{\prime}$ SM process are presented in Figure 4 . The main background process that gives the same final state as excited neutrino signal is multijet neutral current deep inelastic scattering (NC DIS) events. After applying these cuts we obtained the SM background cross-section for the process ep $\rightarrow v^{\star} X \rightarrow e^{-} W^{+} X$ as $\sigma_{B}=0.334 \mathrm{pb}$ for $\sqrt{s}=1.3 \mathrm{TeV}$ and $\sigma_{B}=0.928 \mathrm{pb}$ for $\sqrt{s}=$ $1.98 \mathrm{TeV}$. In order to discriminate the excited neutrino signal we plot the invariant mass distributions for the $\mathrm{eW}$ system for the masses $m^{\star}=400,500,600 \mathrm{GeV}$ at $\sqrt{s}=1.3 \mathrm{TeV}$ and for the masses $m^{\star}=700,800,900 \mathrm{GeV}$ at $\sqrt{s}=1.98 \mathrm{TeV}$ in Figures 5 and 6 , respectively.

We plot the rate of $\sigma_{B+S} / \sigma_{B}$ as a function of excited neutrino mass in Figure 7 to examine the contribution of excited neutrinos to the process $e^{-} q\left(\bar{q}^{\prime}\right) \rightarrow W^{+} e^{-} q^{\prime}(\bar{q})$ and also to investigate the separation of different excited neutrino models. Here $\sigma_{B+S}$ corresponds the cross-section calculated for the presence of excited neutrino (signal) and Standard Model (background) both, and $\sigma_{B}$ is the SM (background) cross-section. In these figures, the separation of spin-1/2, spin-3/2 with $J_{1}$ and spin-3/2 with $J_{2}$ excited neutrinos can be easily seen.

In order to get accessible limits for the excited neutrinos at high energy ep collider, we plot the contours for excited neutrinos with spin-1/2 and spin-3/2. We choose the $W$ boson decay as $W \rightarrow 2 j$. Here we consider the statistical significance:

$$
\mathrm{SS}=\frac{\sigma_{S}}{\sqrt{\sigma_{B}}} \sqrt{L_{\text {int }}} .
$$

Here $L_{\text {int }}$ is the integrated luminosity of the ep collider and we choose $L_{\text {int }}=100 \mathrm{fb}^{-1}$ as the LHeC design luminosity. Our results for the SS are shown in Tables 4 and 5.

For the criteria SS $\geqslant 3$ (95\% C.L.) we plot the $c_{i V}-c_{i A}$ $(i=1,2)$ contour plot for excited spin-3/2 neutrinos for both phenomenological currents and the $f-f^{\prime}$ contour plot for the excited spin-1/2 neutrinos. In Figures 8 and 9, we choose the excited neutrino mass $m^{\star}=400 \mathrm{GeV}$ for the analysis at $\sqrt{s}=1.3 \mathrm{TeV}$ and $m^{\star}=800 \mathrm{GeV}$ for the analysis at $\sqrt{s}=1.98 \mathrm{TeV}$. We see from these figures the allowed regions 

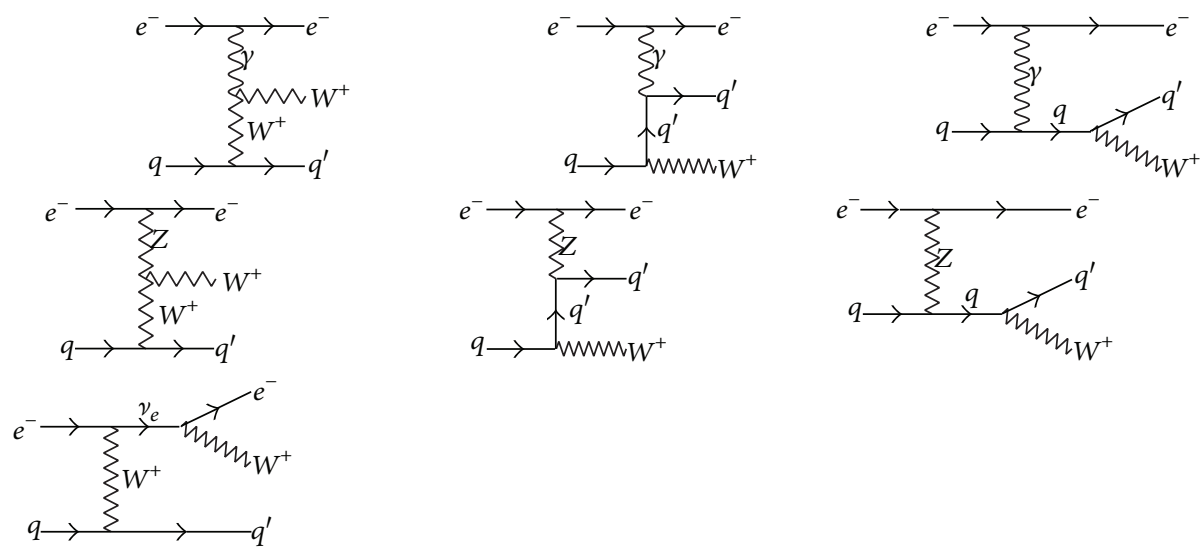

FIGURE 4: Feynman diagrams for the SM background process $e^{-} q \rightarrow e^{-} W^{+} q^{\prime}$.
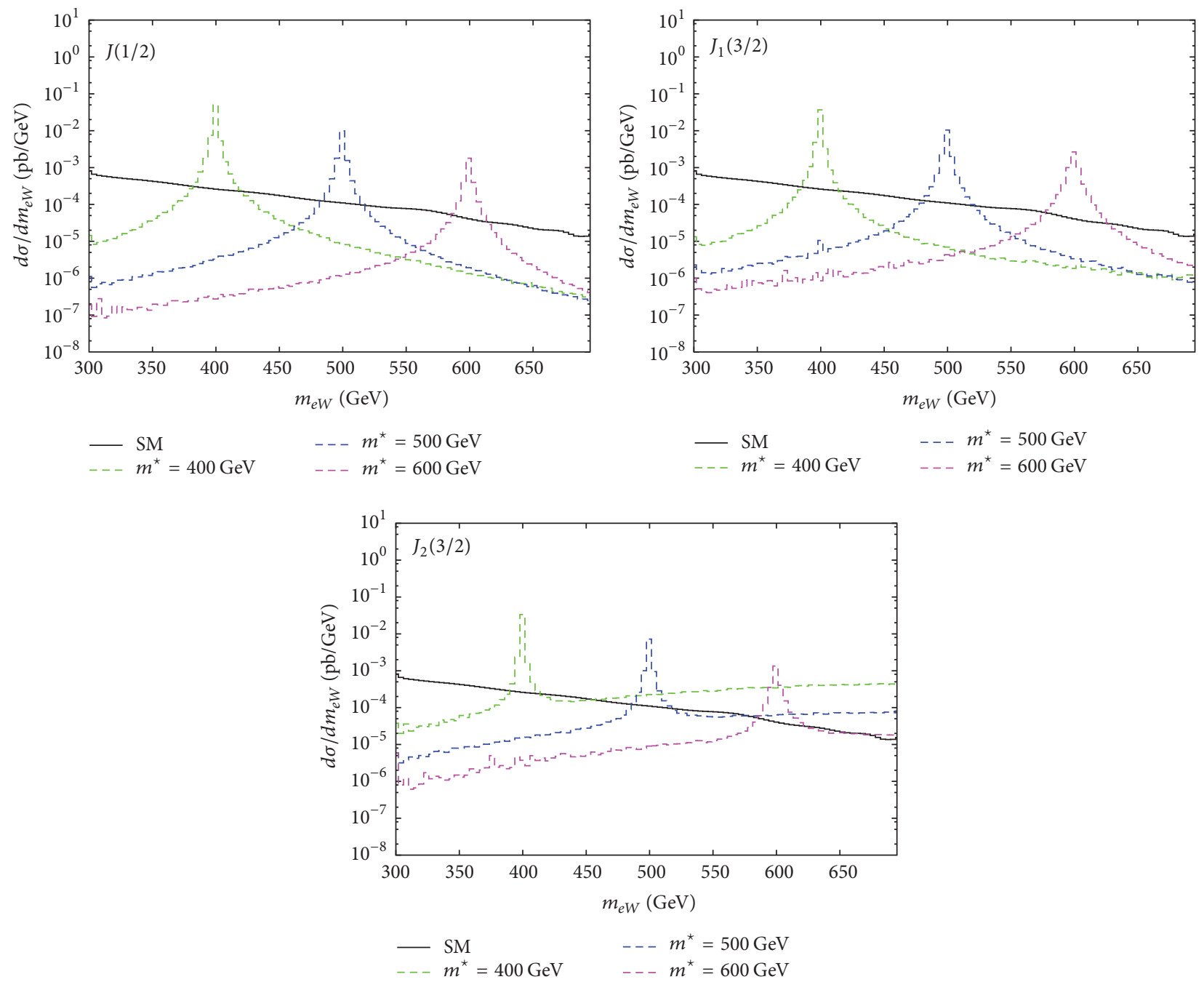

FIGURE 5: Invariant mass distributions of $e W$ system for the single production of excited spin-1/2 for $f=-f^{\prime}=1$ and excited spin-3/2 neutrinos with $J_{1}$ and $J_{2}$ for $c_{i V}=c_{i A}=0.5(i=1,2)$ at $\sqrt{s}=1.3 \mathrm{TeV}$. 
TABLE 3: Branching ratios and total decay width of excited spin-3/2 neutrinos with $J_{2}$. Here it is taken as $c_{2 V}=c_{2 A}=0.5$ and $\Lambda=m^{\star}$.

\begin{tabular}{lcccc}
\hline$m^{\star}(\mathrm{GeV})$ & $\Gamma(\mathrm{GeV})$ & $\% \mathrm{BR}\left(\nu^{\star} \rightarrow \nu \gamma\right)$ & $\% \mathrm{BR}\left(\nu^{\star} \rightarrow \nu Z\right)$ & $\% \mathrm{BR}\left(\nu^{\star} \rightarrow e W\right)$ \\
\hline 300 & 0.55 & 8.8 & 38.4 & 52.8 \\
500 & 2.71 & 3.0 & 41.8 & 55.3 \\
750 & 9.31 & 1.3 & 42.7 & 56.0 \\
1000 & 22.21 & 0.7 & 43.0 & 56.2 \\
1500 & 75.26 & 0.3 & 43.3 & 56.4 \\
2000 & 178.7 & 0.2 & 43.4 & 56.5 \\
2500 & 349.2 & 0.1 & 43.4 & 56.5 \\
3000 & 603.6 & 0.1 & 43.4 & 56.5 \\
\hline
\end{tabular}
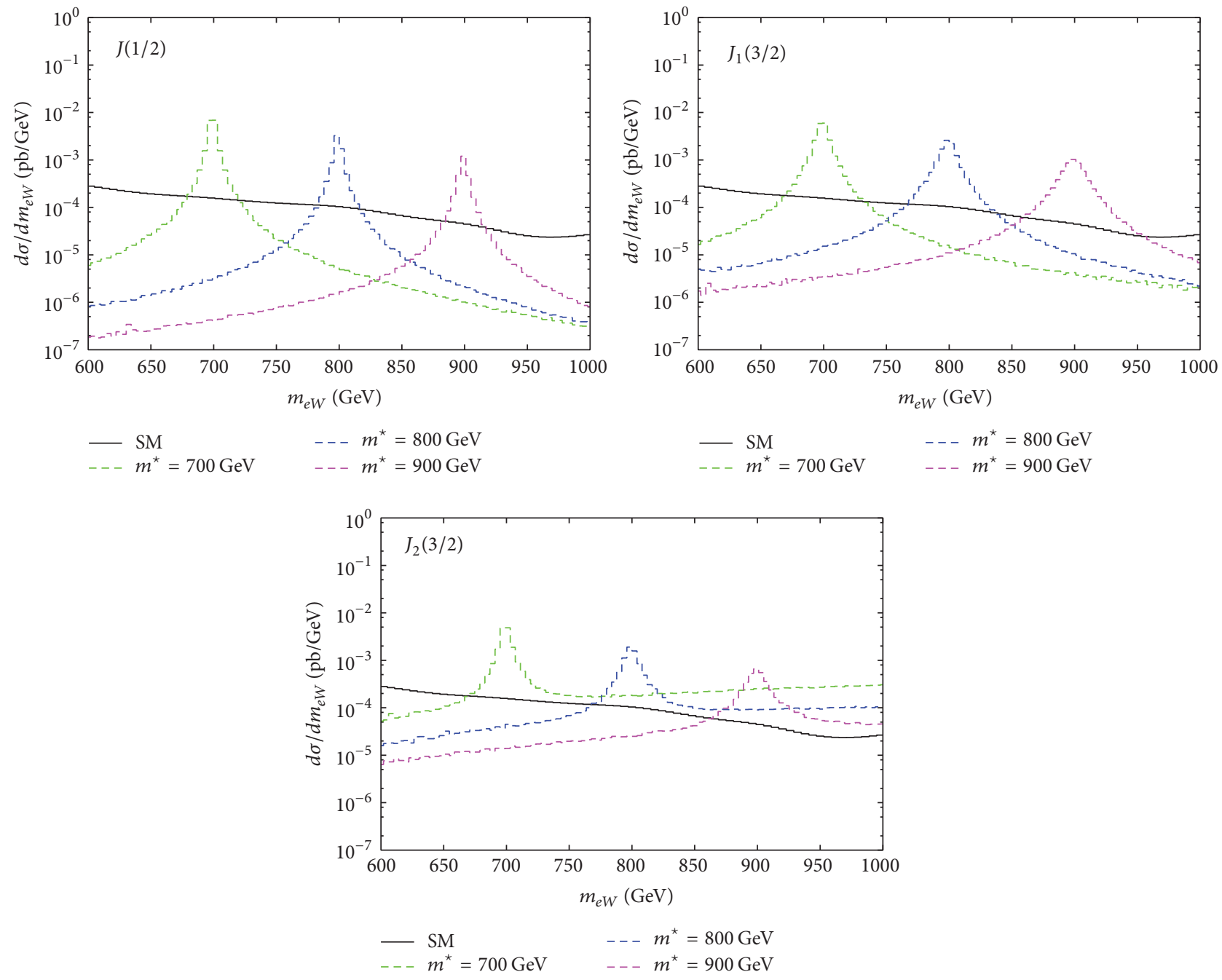

FIGURE 6: Invariant mass distributions of $e W$ system for the single production of excited spin-1/2 for $f=-f^{\prime}=1$ and excited spin-3/2 neutrinos with $J_{1}$ and $J_{2}$ for $c_{i V}=c_{i A}=0.5(i=1,2)$ at $\sqrt{s}=1.98 \mathrm{TeV}$.

for the $c_{i V}-c_{i A}(i=1,2)$ and $f-f^{\prime}$ couplings for the masses $m^{\star}=400 \mathrm{GeV}$ at $\sqrt{s}=1.3 \mathrm{TeV}$ and $m^{\star}=800 \mathrm{GeV}$ at $\sqrt{s}=1.98 \mathrm{TeV}$. The values which we chose in our calculations for the coupling parameters $\left(c_{i V}=c_{i A}=0.5\right.$ for the excited spin-3/2 neutrinos and $f=-f^{\prime}=1$ for the excited spin-1/2 neutrinos) are compatible with the contour plots.

\section{Conclusion}

We searched for the excited spin-3/2 neutrino signal at lepton-hadron collider $\mathrm{LHeC}$ for two different centers of mass energies. We used two different phenomenological currents for the spin-3/2 excited neutrinos, and we used the same value 


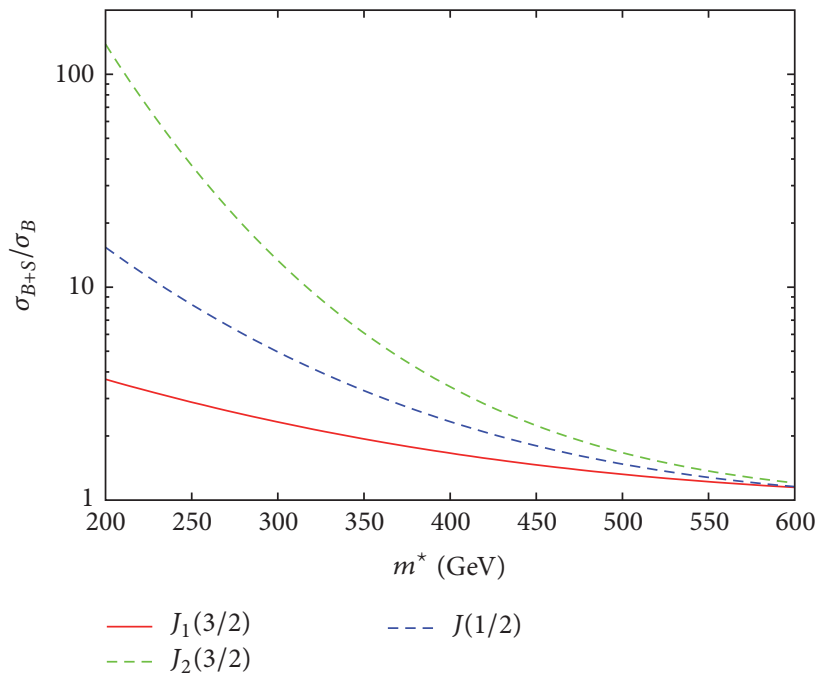

(a)

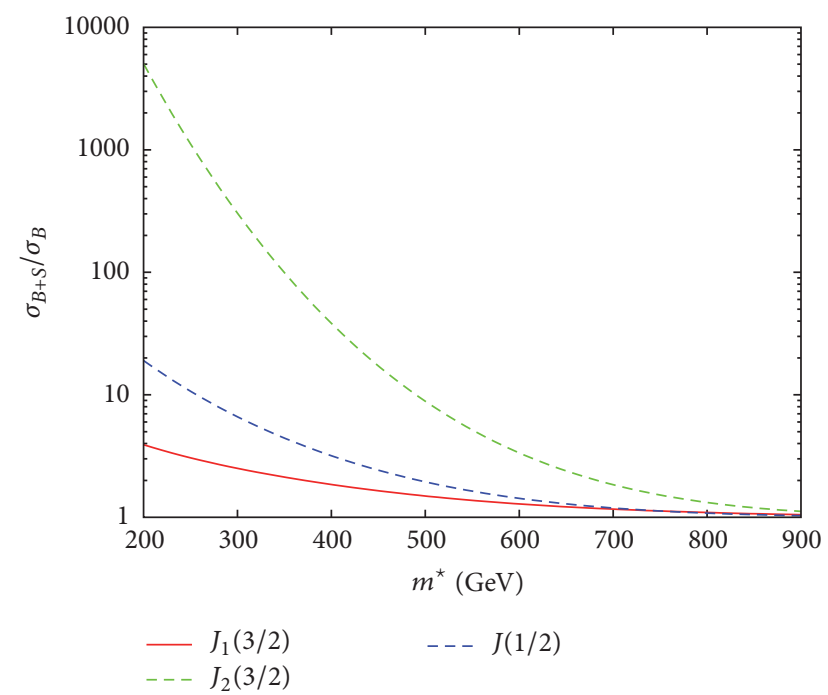

(b)

Figure 7: $\sigma_{B+S} / \sigma_{B}-m^{\star}$ plots for $\sqrt{s}=1.3 \mathrm{TeV}(\mathrm{a})$ and $\sqrt{s}=1.98 \mathrm{TeV}(\mathrm{b}) .\left(f=-f^{\prime}=1\right.$ for the spin-1/2 and $c_{i V}=c_{i A}=0.5(i=1,2)$ for the spin-3/2.)
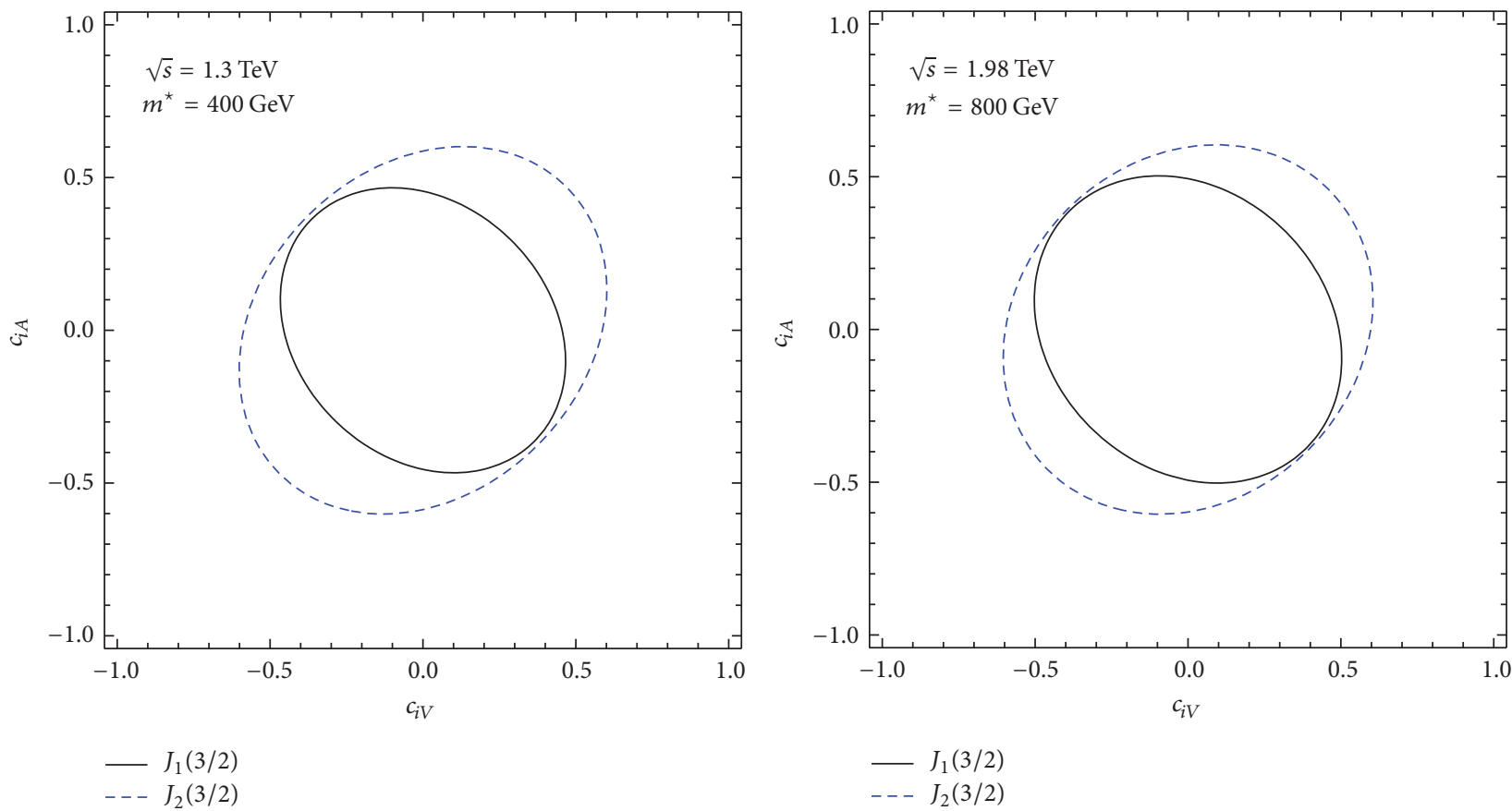

FIGURE 8: Contour plots for excited spin-3/2 neutrinos for the $J_{1}$ and $J_{2}$.

of $c_{i V}, c_{i A}(i=1,2)$ couplings. Since there is no theoretical prediction for the single production of excited neutrinos and the effective currents have unknown couplings, we did not consider the interference between the currents. A more detailed calculation shows an important parameter space in which the interference terms could be important.

We also deal with the spin-1/2 excited neutrinos for comparison. Our analysis shows that the spin- $1 / 2$ and spin- $3 / 2$ excited neutrino signals discrimination is apparent at next $e p$ colliders. Here we only take into account the effective currents describing the gauge interactions of excited and standard particles. It is possible to include the contact interactions which may enlarge the mass and coupling limits.

It is possible to search for single production of excited spin-3/2 neutrinos at the LHC but it has smaller cross-section than $\mathrm{LHeC}$. Therefore, the potential of $\mathrm{LHeC}$ is better than LHC to determine the limits on couplings of excited spin-3/2 neutrinos. 

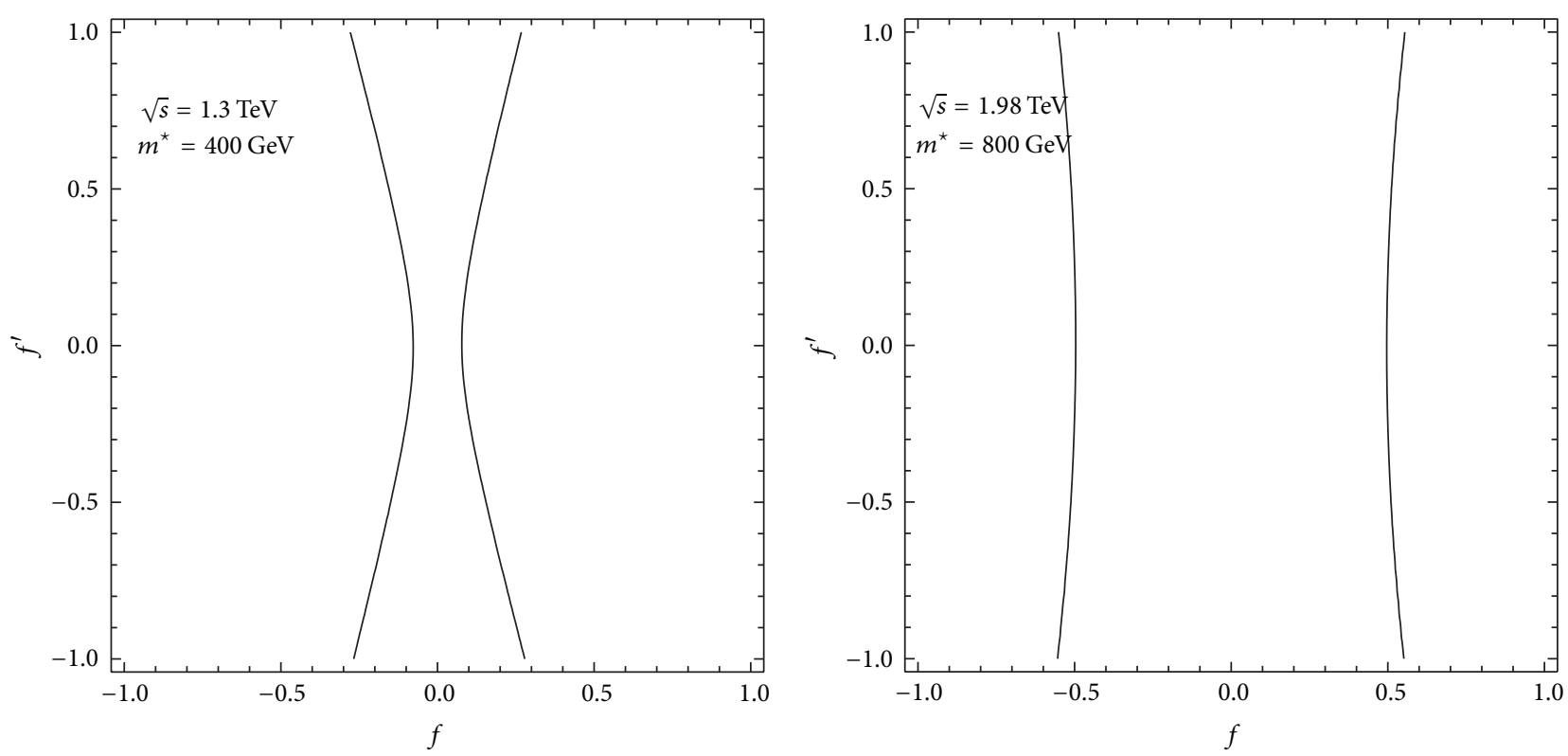

FIgURE 9: Contour plots for excited spin-1/2 neutrinos.

TABLE 4: Statistical significance SS for ep collider with $\sqrt{s}=1.3 \mathrm{TeV}$ for excited spin-1/2 neutrinos and excited spin-3/2 neutrinos with $J_{1}$ and $J_{2}$.

\begin{tabular}{lccc}
\hline$m^{\star}(\mathrm{GeV})$ & $\mathrm{SS}(J(1 / 2))$ & $\mathrm{SS}\left(J_{1}(3 / 2)\right)$ & $\mathrm{SS}\left(J_{2}(3 / 2)\right)$ \\
\hline 400 & 110.2 & 75.4 & 135.6 \\
500 & 25.5 & 30.7 & 30.0 \\
600 & 5.5 & 11.9 & 7.9 \\
700 & 1.0 & 4.2 & 2.2 \\
\hline
\end{tabular}

TABLE 5: Statistical significance SS for ep collider with $\sqrt{s}=1.98 \mathrm{TeV}$ for excited spin-1/2 neutrinos and excited spin-3/2 neutrinos with $J_{1}$ and $J_{2}$.

\begin{tabular}{lccc}
\hline$m^{\star}(\mathrm{GeV})$ & $\mathrm{SS}(J(1 / 2))$ & $\mathrm{SS}\left(J_{1}(3 / 2)\right)$ & $\mathrm{SS}\left(J_{2}(3 / 2)\right)$ \\
\hline 600 & 56.3 & 51.0 & 235.9 \\
700 & 22.4 & 28.0 & 76.5 \\
800 & 8.8 & 15.1 & 28.9 \\
900 & 3.3 & 8.04 & 12.0 \\
1000 & 1.2 & 4.2 & 5.3 \\
\hline
\end{tabular}

Excited neutrinos with different spins would manifest themselves in three families. Here, we only investigated the excited electron neutrino. It is also possible to make the same analysis for excited muon neutrinos. Single production of excited muon neutrinos is possible at muon-hadron colliders. Physics of $\mu p$ colliders was studied in [54]. One can find the main parameters of FCC-based $\mu p$ collider in $[43,55]$.

\section{Competing Interests}

The authors declare that they have no competing interests.

\section{References}

[1] H. Terazawa, Y. Chikashige, and K. Akama, "Unified model of the Nambu-Jona-Lasinio type for all elementary-particle forces," Physical Review D, vol. 15, no. 2, pp. 480-487, 1977.

[2] Y. Ne'eman, "Primitive particle model," Physics Letters B, vol. 82, no. 1, pp. 69-70, 1979.

[3] H. Terezawa, M. Yasuè, K. Akama, and M. Hayashi, "Observable effects of the possible sub-structure of leptons and quarks," Physics Letters B, vol. 112, no. 4-5, pp. 387-392, 1982.

[4] F. M. Renard, "Excited quarks and new hadronic states," Il Nuovo Cimento A, vol. 77, no. 1, pp. 1-20, 1983.

[5] E. J. Eichten, K. D. Lane, and M. E. Peskin, "New tests for quark and lepton substructure," Physical Review Letters, vol. 50, no. 11, pp. 811-814, 1983.

[6] A. de Rújula, L. Maiani, and R. Petronzio, "Search for excited quarks," Physics Letters B, vol. 140, no. 3-4, pp. 253-258, 1984.

[7] J. Kühn and P. M. Zerwas, "Excited quarks and leptons," Physics Letters B, vol. 147, no. 1-3, pp. 189-196, 1984.

[8] O. Çakır and A. Ozansoy, "Single production of excited spin-3/2 neutrinos at linear colliders," Physical Review D, vol. 79, no. 5, Article ID 055001, 2009.

[9] J. L. Leite Lopes, J. A. Martins Simoes, and D. Spehler, "Production and decay properties of possible spin 32 leptons," Physics Letters B, vol. 94, no. 3, pp. 367-372, 1980.

[10] J. Leite Lopes, J. A. Martins Simoes, and D. Spehler, "Possible spin-3/2 quarks and scaling violations in neutrino reactions," Physical Review D, vol. 23, no. 3, pp. 797-799, 1981.

[11] J. Leite Lopes, D. Spehler, and J. A. Martins Simoes, "Weak interactions involving spin-3/2 leptons," Physical Review D, vol. 25, no. 7, pp. 1854-1859, 1982.

[12] Y. Tosa and R. E. Marshak, "Exotic fermions," Physical Review $D$, vol. 32, no. 3, pp. 774-780, 1985.

[13] O. J. P. Éboli, E. M. Gregores, J. C. Montero, S. F. Novaes, and D. Spehler, "Excited leptonic states in polarized $e^{-} \gamma$ and $e^{+} e^{-}$ collisions," Physical Review D, vol. 53, no. 3, pp. 1253-1263, 1996. 
[14] D. Z. Freedman, P. van Nieuwenhuizen, and S. Ferrara, "Progress toward a theory of supergravity," Physical Review. D, vol. 13, no. 12, pp. 3214-3218, 1976.

[15] M. Sahin, "Resonant production of Spin-3/2 color octet electron at the LHeC," Acta Physica Polonica B, vol. 45, no. 9, pp. 18111831, 2014.

[16] J. F. Nieves, "Electromagnetic properties of spin-3/2 Majorana particles," Physical Review D, vol. 88, no. 3, Article ID 036006, 2013.

[17] D. A. Dicus, D. Karabacak, S. Nandi, and S. K. Rai, "Search for spin-3/2 quarks at the Large Hadron Collider," Physical Review D, vol. 87, no. 1, Article ID 015023, 2013.

[18] W. J. Stirling and E. Vryonidou, "Effect of spin-3/2 top quark excitation on $t \bar{t}$ production at the LHC," JHEP, vol. 1201, article 055, 2012.

[19] M. Lindner, F. S. Queiroz, W. Rodejohann, and C. E. Yaguna, "Left-right symmetry and lepton number violation at the Large Hadron electron Collider," Journal of High Energy Physics, vol. 2016, article 140, 2016.

[20] F. Boudjema and A. Djouadi, "Looking for the LEP at LEP. The excited neutrino scenario," Physics Letters B, vol. 240, no. 3-4, pp. 485-491, 1990.

[21] B. Adeva, O. Adriani, M. Aguilar-Benitez et al., "Search for excited neutrinos from $Z^{0}$ decays," Physics Letters B, vol. 252, no. 3, pp. 525-532, 1990.

[22] D. Decamp, B. Deschizeaux, C. Goy et al., "Search for excited neutrinos in Z decay," Physics Letters B, vol. 250, no. 1-2, pp. 172182,1990

[23] O. J. P. Eboli, S. M. Lietti, and P. Mathews, "Excited leptons at the CERN large Hadron collider," Physical Review D, vol. 65, no. 7, Article ID 075003, 2002.

[24] A. Belyaev, C. Leroy, and R. Mehdiyev, "Production of excited neutrinos at the LHC," The European Physical Journal C, vol. 41, supplement 2, pp. 1-10, 2005.

[25] O. Çakır, İ. T. Çakır, and Z. Kırca, "Single production of excited neutrinos at future $\mathrm{e}^{+} \mathrm{e}^{-}, e p$ and $p p$ colliders," Physical Review D, vol. 70, no. 7, Article ID 075017, 2004.

[26] A. Belyaev and E. Boos, "Excited neutrinos at the next linear colliders," Physics of Atomic Nuclei, vol. 56, pp. 1447-1454, 1993.

[27] A. Belyaev and E. Boos, "Excited neutrinos at the next linear colliders," Physics of Atomic Nuclei, vol. 56, no. 11, pp. 5-15, 1993.

[28] A. Belyaev, E. Boos, and A. Pukhov, "Study of excited neutrino production in $e^{+} e^{-}, \gamma e$ and $\gamma \gamma$ collisions at TeV energies," Physics Letters B, vol. 296, no. 3-4, pp. 452-457, 1992.

[29] M. Köksal, "Analysis of excited neutrinos at the CLIC," International Journal of Modern Physics A, vol. 29, no. 24, Article ID 1450138, 2014.

[30] R. Walsh and A. J. Ramalho, "Virtual excited neutrino exchange in W boson pair production," Physical Review D, vol. 67, no. 9, Article ID 097702, 2003.

[31] M. M. Reynoso, I. Romero, and O. A. Sampayo, "Excited neutrino production by ultrahigh energy neutrinos traversing the Earth," Physical Review D, vol. 86, no. 11, Article ID 113012, 2012.

[32] S. C. İnan and M. Köksal, "The impact of excited neutrinos on $\nu \bar{v} \rightarrow \gamma \gamma$ process," Advances in High Energy Physics, vol. 2012, Article ID 571874, 8 pages, 2012.

[33] P. Achard, O. Adriani, M. Aguilar-Benitez et al., "Search for excited leptons at LEP," Physics Letters B, vol. 568, no. 1-2, pp. 23-34, 2003.
[34] F. D. Aaron et al., "A search for excited neutrinos in $\mathrm{e}^{-} \mathrm{p}$ collisions at HERA," Physics Letters B, vol. 663, no. 5, pp. 382389, 2008.

[35] G. Aad, B. Abbott, J. Abdallah et al., "Search for new phenomena in events with three or more charged leptons in $p p$ collisions at $\sqrt{s}=8 \mathrm{TeV}$ with the ATLAS detector," Journal of High Energy Physics, vol. 2015, article 138, 2015.

[36] S. R. Choudhury, R. G. Ellis, and G. C. Joshi, "Limits on excited spin-3/2 leptons," Physical Review D, vol. 31, no. 9, pp. 23902392, 1985.

[37] F. M. L. Almeida, J. H. Lopes, J. A. Martins Simões, and A. J. Ramalho, "Production and decay of single heavy spin-3/2 leptons in high energy electron-positron collisions," Physical Review D, vol. 53, no. 7, article 3555, 1996.

[38] J. L. Abelleira Fernandez, C. Adolphsen, A. N. Akay et al., "A large hadron electron collider at CERN: report on the physics and design concepts for machine and detector," Journal of Physics G: Nuclear and Particle Physics, vol. 39, no. 7, Article ID 075001, 2012.

[39] O. Brüning and M. Klein, "The large hadron electron collider," Modern Physics Letters A, vol. 28, no. 16, Article ID 1330011, 2013.

[40] P. Newman and A. Stasto, "Dig deeper," Nature Physics, vol. 9, no. 8, pp. 448-450, 2013.

[41] T. N. Trinh and E. Sauvan, "Single production of excited leptons at the LHeC," CERN Internal Note CERN-LHeC-Note-2010-011 PHY, 2011, https://cds.cern.ch/record/1354237.

[42] Website of FCC Study, https://fcc.web.cern.ch.

[43] Y. C. Acar, U. Kaya, B. B. Oner, and S. Sultansoy, "FCC based ep and $\mu$ p colliders," https://arxiv.org/abs/1510.08284.

[44] P. L. Csonka and J. Rees, "A device to produce high center-ofmass energy e+e collisions-accelerator beam colliding with a stored beam," Nuclear Instruments and Methods, vol. 96, no. 1, pp. 149-155, 1971.

[45] S. Sultansoy, "Four ways to TeV scale," Turkish Journal of Physics, vol. 22, no. 7, pp. 575-594, 1998.

[46] S. Sultansoy, "Linac ring type colliders: second way to $\mathrm{TeV}$ scale," The European Physical Journal C, vol. 33, supplement 1, pp. s1064-s1066, 2004.

[47] K. Hagiwara, D. Zeppenfeld, and S. Komamiya, "Excited lepton production at LEP and HERA," Zeitschrift für Physik C, vol. 29, no. 1, pp. 115-122, 1985.

[48] F. Boudjema, A. Djouadi, and J. L. Kneur, "Excited fermions at $e^{+} e^{-}$and $\mathrm{eP}$ colliders," Zeitschrift für Physik C Particles and Fields, vol. 57, no. 3, pp. 425-449, 1993.

[49] U. Baur, M. Spira, and P. M. Zerwas, "Excited-quark and -lepton production at hadron colliders," Physical Review D, vol. 42, no. 3, pp. 815-824, 1990.

[50] W. Rarita and J. S. Schwinger, "On a theory of particles with halfintegral spin," Physical Review, vol. 60, no. 1, p. 61, 1941.

[51] A. Belyaev, N. D. Christensen, and A. Pukhov, "CalcHEP 3.4 for collider physics within and beyond the Standard Model," Computer Physics Communications, vol. 184, no. 7, pp. 17291769, 2013.

[52] A. Pukhov, "CalcHEP 2.3: MSSM, structure functions, event generation, batchs, and generation of matrix elements for other packages," https://arxiv.org/abs/hep-ph/0412191.

[53] A. Pukhov, E. Boos, M. Dubinin et al., "CompHEP—a package for evaluation of Feynman diagrams and integration over multi-particle phase space. User's manual for version 33," https://arxiv.org/abs/hep-ph/9908288. 
[54] I. F. Ginzburg, "Physics at future e p, gamma p (linac-ring) and mu p colliders," Turkish Journal of Physics, vol. 22, pp. 607-610, 1998.

[55] Y. Acar, A. N. Akay, S. Beser et al., "FCC based LeptonHadron and Photon-Hadron colliders: luminosity and physics," https://arxiv.org/abs/1608.02190. 

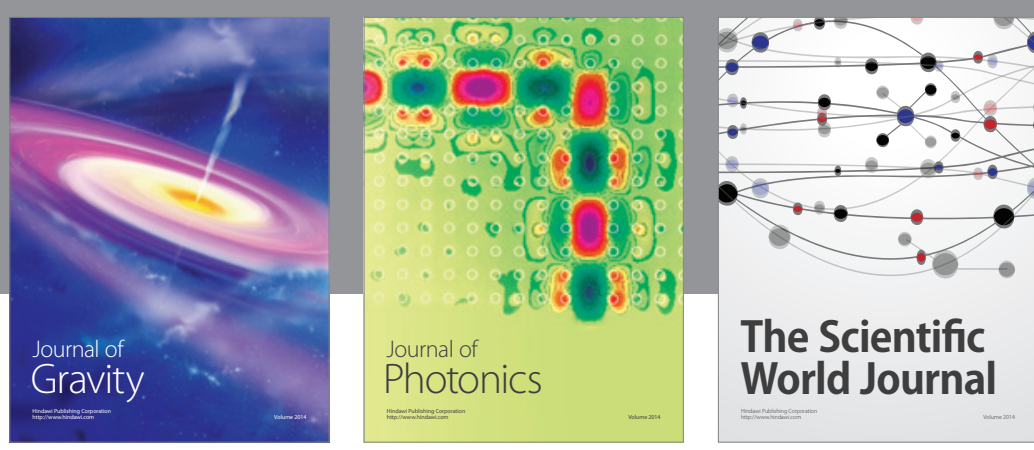

The Scientific World Journal
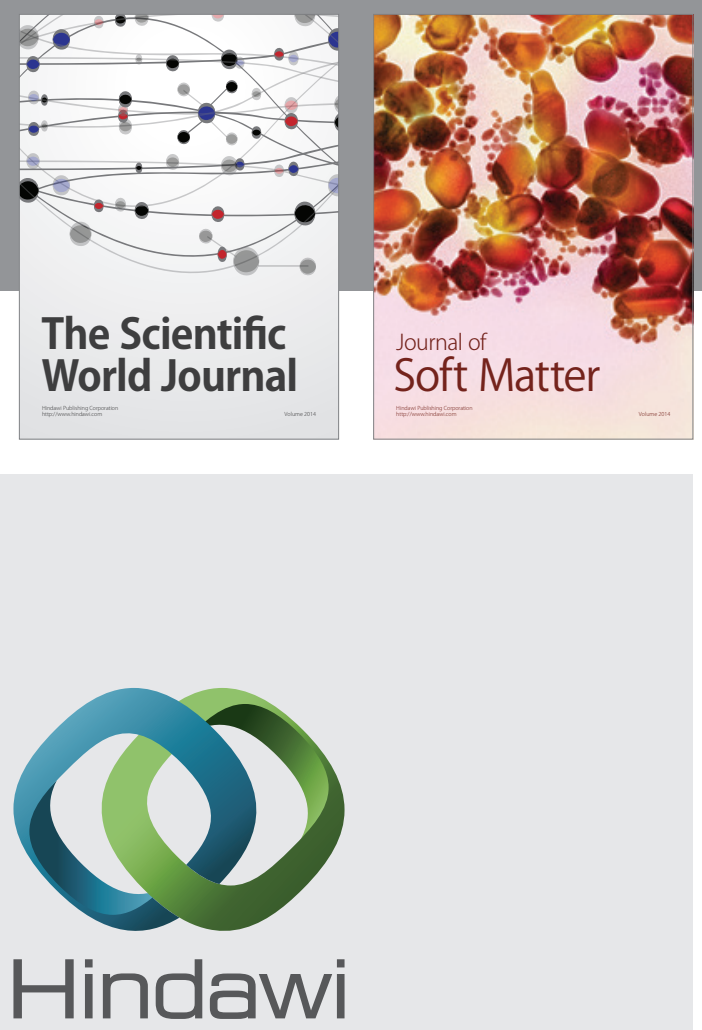

Submit your manuscripts at

http://www.hindawi.com

nternational Journal of

Statistical Mechanics
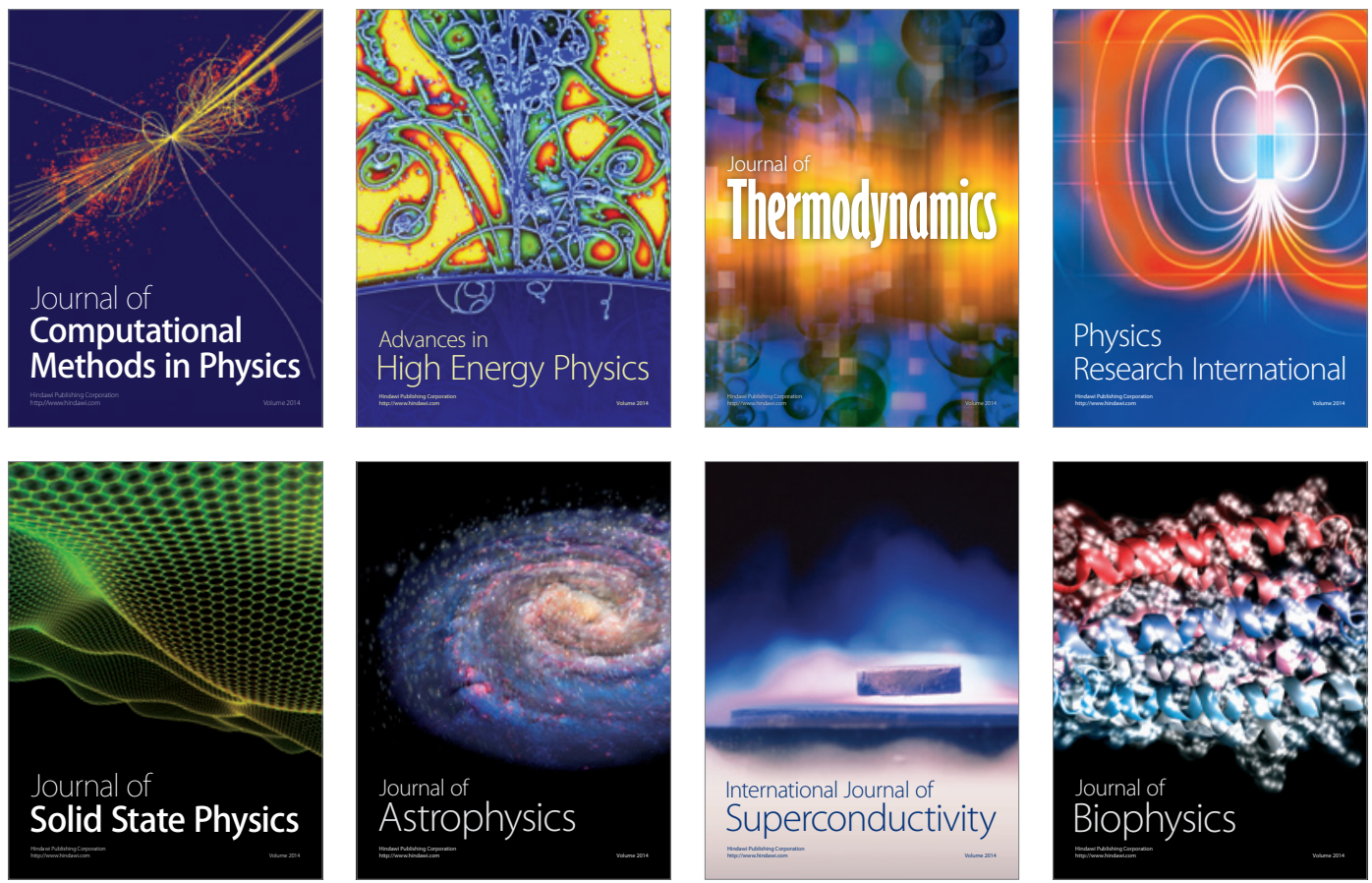
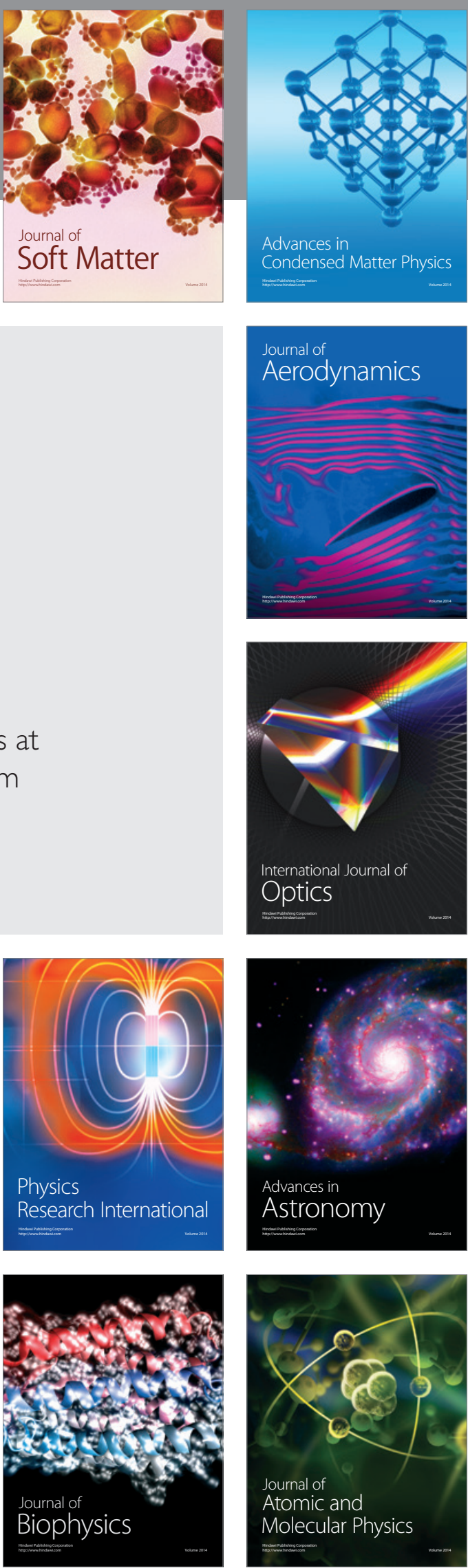